\title{
Discussion on Feasibility and Safety of Pulmonary Segmentectomy under Total Thoracoscope in Treatment of Peripheral Early Lung Cancer and Pulmonary Metastasis Tumor
}

\author{
Tao Zhang, Yuanfu Wei* \\ Department of Cardiothoracic Vascular Surgery, Renmin Hospital, Hubei University of Medicine, \\ Shiyan, Hubei, China, 442000 \\ *Corresponding author E-Mail:hubeiwenbo@qq.com \\ Address: Chaoyang District Road No. 39, Shiyan City, Hubei Province, P.R.China, 442000
}

Keywords: peripheral lung cancer; thoracoscope; pulmonary segmentectomy; prognosis

\begin{abstract}
Objective: to discuss feasibility and safety of pulmonary segmentectomy under total thoracoscope in treatment of peripheral early lung cancer and pulmonary metastasis tumor. Method: the clinical data of 25 patients with peripheral early lung cancer and pulmonary metastasis tumor who received pulmonary segmentectomy under total thoracoscope in our hospital from February 2016 to February 2018 were retrospectively analyzed. Results: the operation was completed successfully for all 25 patients. Intraoperative bleeding volume $(105.6 \pm 6.2) \mathrm{ml}$, duration of operation $(137.6 \pm 8.0) \mathrm{min}$, duration of postoperative drainage $(2.6 \pm 0.7) \mathrm{d}$, duration of hospitalization (7.1 \pm 1.3$) \mathrm{d}$. Pulmonary atelectasis happened to 3 patients after the operation. After the corresponding treatment was given, the symptom disappeared. Bronchopleural fistula, respiratory failure, infection of incisional wound and other severe complications were not found. The wound healing was good, and scar tissue was not found. During 2-year postoperative follow-up visit, tumor metastasis or relapse was not seen. Conclusion: pulmonary segmentectomy under total thoracoscope has high safety in treatment of peripheral early lung cancer and pulmonary metastasis tumor. Besides, it has good feasibility. Therefore, it deserves to be promoted.
\end{abstract}

Lung cancer is a common clinical disease, and its morbidity ad death rate rank top among all tumors. Lung cancer is classified into two types: small cell lung cancer and non-small cell lung cancer. The number of patients with non-small cell lung cancer accounts for about $85 \%$ of total number ${ }^{[1-2]}$. The treatment of non-small cell lung cancer in the early stage mainly adopts excision. Operative treatment involves traditional thoracotomy treatment and pulmonary segmentectomy under total thoracoscope. In this study, feasibility and safety of pulmonary segmentectomy under total thoracoscope in treatment of peripheral early lung cancer and pulmonary metastasis tumor were discussed. 


\section{Data and Method}

\subsection{Clinical data}

There were 25 patients with peripheral early lung cancer and pulmonary metastasis tumor who received pulmonary segmentectomy under total thoracoscope in our hospital from February 2016 to February 2018, including 12 male patients and 13 female patients, with the age of 19-74 and average age of $55.4 \pm 1.3$. 7 of them were diagnosed as primary non-small cell lung cancer, 3 of them were diagnosed as pulmonary metastasis tumor ( 2 with choriocarcinoma pulmonary metastasis and 1 with breast cancer with lung metastasis). 15 of them were diagnosed as benign tumor ( 2 with sclerosing hemangioma, 3 with inflammatory pseudotumor, 3 with pulmonary tuberculoma and 7 with hamartoma). All patients agreed to receive pulmonary segmentectomy under total thoracoscope.

\subsection{Method}

All patients received pulmonary segmentectomy under total thoracoscope. Double lumen intubation was used for general anesthesia. The patients adopted $90^{\circ}$ recumbent position, with one-lung ventilation. The cushion was put under the chest, and the operating table was slightly lifted to keep the head higher and the feet lower. Thoracoscope incision was carried out. $1 \mathrm{~cm}$ incision was made between the midaxillary line and the $7^{\text {th }}$ rib of patients. The thoracoscope was put through the incision. The main operation hole is made, and $3 \mathrm{~cm}$ incision was made between the anterior axillary line and the $4^{\text {th }}$ rib of patients. Then, the auxiliary operation hole is made, and $2 \mathrm{~cm}$ incision was made between the infrascapular line and the $9^{\text {th }}$ rib of patients. Thoracic mucosa adhesion and diseased region of patients' thoracic cavity were understood in detail through the video-assisted thoracoscope. The pleura around the pulmonary segment and nearby leaf lobes were opened. For the patients with the high probability of tumor, the sections were frozen after mediastinal lymph nodes sampling and hilar lymph nodes sampling were finished. Different measures were taken according to lung fissure development conditions. For those with good development, pulmonary artery, pulmonary vein and bronchus were handled successively. For those with poor development, dissection was conducted for the root of pulmonary segment layer by layer along the same direction. Endoscope cutting anastomat was used to handle inter-segment tissue, bronchus, blood vessel in pulmonary segment and lung fissure. The knot pusher was applied for double ligation of distal end and proximal end to complete the treatment of fine vessels. The excision number and parts of intraoperative pulmonary segments are shown in Table 1. During handling primary cancer, the distance between incisal edge and tumor shall be more than $2 \mathrm{~cm}$. If necessary, near lung tissues can be cut off ${ }^{[3-5]}$.

Table 1

\begin{tabular}{lccc}
\hline \multicolumn{1}{c}{ Operation } & Part & Left & Right \\
\hline $\begin{array}{l}\text { Excision of multiple } \\
\text { pulmonary segments }\end{array}$ & Rear + front + sharp & 5 & 0 \\
$\begin{array}{l}\text { Excision of double } \\
\text { pulmonary segments }\end{array}$ & Tongue & 6 & 0 \\
$\begin{array}{l}\text { Excision of single } \\
\text { pulmonary segment }\end{array}$ & Front & 0 & 6 \\
- & & & \\
- & Rear & 0 & 2 \\
\hline
\end{tabular}


After the operation was finished, the drainage tube was indwelled. After postoperative narcotism disappeared, the bronchial tube could be removed, and patients could be sent to ICU. Chest radiography reexamination was conducted $24 \mathrm{~h}$ after the operation, and the results showed that there was no pneumothorax and hydrops; lung tension was good; the drainage tube was removed when the amount of thoracic cavity drainage was $50 \mathrm{ml}$. The patients were encouraged to get out of bed as early as possible to recover lung functions. When no abnormal conditions were found in the laboratory examination and patients' lung function recovered well, they could leave hospital. Postoperative follow-up visit was implemented and follow-up treatment was given for the patients.

\section{Results}

\subsection{Operation conditions and operation indicators}

The operation was completed successfully for all 25 patients. No patient died. Among the 7 patients with early primary non-small cell lung cancer, 1 showed positive incisal edge of lung fissure in pathological examination of frozen sections, and the patient received pulmonary lobectomy under thoracoscope. The patient's amount of intraoperative bleeding was $(105.6 \pm 6.2) \mathrm{ml}$, duration of operation was $(137.6 \pm 8.0) \mathrm{min}$, postoperative drainage duration was $(2.6 \pm 0.7) \mathrm{d}$, and duration of hospital stay (7.1 \pm 1.3$)$ d.

\subsection{Complications and prognoses}

Pulmonary atelectasis happened to 3 paints after the operation. After sputum aspiration with the bronchoscope was conducted, the symptom disappeared. Bronchopleural fistula, respiratory failure, infection of incisional wound and other severe complications were not found. The wound healing was good, and scar tissue was not found. During 2-year postoperative follow-up visit, tumor metastasis or relapse was not seen.

\section{Discussion}

Lung cancer mainly includes squamous carcinoma, large cell carcinoma and adenocarcinoma, and it is one of common pulmonary diseases. Lung cancer threatens patients' life safety while affecting patients' living quality and health. Thus, when patients are diagnosed as early stage of lung cancer, effective and timely early treatment must be given in time to prevent further development of the disease. For the treatment means of early stage of lung cancer, pulmonary segmentectomy is the most common treatment method ${ }^{[6]}$. Pulmonary segmentectomy refers to excision of patients' one or more pulmonary segment tissues with pathological tissues, and the operation is completed mostly through one-way handling of bronchus, artery and vein on pulmonary segments. The traditional treatment operation of pulmonary segmentectomy is thoracotomy which has many defects such as large trauma, difficulty in reaching precise lung tissue excision (which may lead to excision of healthy lung tissues), slow recovery speed and many complications. The operation under thoracoscope belongs to minimally invasive surgery. The thoracoscope can provide the operators with surgery field and the operators can carry out the operation under the help of video image. In the early stage, it is only used treat pleural diseases. With the development of modern medical technology, thoracoscope starts to be applied in surgical operation field. Its principle is as follows: excision of segmental bronchi, axillary structure, artery and vein on the pulmonary segments under the assistance of total thoracoscope. Since the thoracoscope is used to provide surgical field, the operators do not need to use incision retractor and thoracotomy cutter, which contributes to reducing amount of intraoperative bleeding, avoids 
incision suture and thus can shorten the duration of operation. Pulmonary segmentectomy under total thoracoscope has the characteristics of clear surgical field, minimal invasion, fast postoperative recovery and high excision precision, so it is an important technique. The thoracoscope can provide operators with the good surgical field so that the operators can accurately and minimally invasively cut off the diseased lung tissues. Besides, the lung tissues can be reserved as far as possible, which contributes to protecting patients' respiratory function.

The application scope of pulmonary segmentectomy under total thoracoscope involves invasive carcinoma, slightly invasive adenocarcinoma and carcinoma in situ. During handling invasive carcinoma, the common standard treatment measure is that lymph node dissection or sampling is given after anatomical excision of patients' pulmonary lobe. Dissection of 6 lymph nodes (3 groups of mediastinal lymph nodes) in each group is recommended currently, or lymph node sampling is conducted. No matter which method is adopted, sampling or dissection of subcarinal lymph nodes is required. During treatment of slightly invasive adenocarcinoma, sampling or dissection of subcarinal lymph nodes is not needed. When the focus is in the internal circumference, pulmonary segmentectomy under total thoracoscope can be implemented. When the focus is in the periphery, pulmonary segmentectomy or wedge-shaped pneumonectomy can be applied. During treatment of carcinoma in situ, sampling or dissection of subcarinal lymph nodes is not needed. Pulmonary segmentectomy under total thoracoscope, pulmonary sub-segmentectomy or wedge-shaped pneumonectomy can be applied ${ }^{[7]}$.

For the mode of pulmonary segmentectomy under total thoracoscope, there is single-hole pulmonary segmentectomy under total thoracoscope and multi-hole pulmonary segmentectomy under total thoracoscope according to the differences of hole number. Since pulmonary segmentectomy under singlehole thoracoscope only requires one hole, only 1 intercostal nerve is damaged, which can to a larger degree reduce incision pain degree. The unique surgical field contributes to fully exposing superior mediastinum so as to form the surgical field similar to thoracotomy. However, the thoracoscope and operating apparatus share the one hole in the operation process of pulmonary segmentectomy under singlehole thoracoscope, apparatus interference possibility exists. The operating apparatus needs to go in and out many times, lung exposure effect will be affected. Where hemorrhage during operation appears or thoracic adhesion is severe, the operation difficulty of single-hole thoracoscope may be large, and hemorrhage during operation is the main risk of e pulmonary segmentectomy under single-hole thoracoscope. When hemorrhage during operation happens, hemostasis by compression shall be adopted first, and patients' hemorrhage degree shall be evaluated. When slight bleeding happens to pulmonary artery branch, titanium clamp may be used to stop bleeding or suturing is conducted under the single hole. When it is hard to operate under the single hole, two-hole or three-hole thoracoscope shall be used. When bleeding degree is severe, thoracotomy shall be applied. Pulmonary segmentectomy under multihole thoracoscope requires multiple holes, so it enhances patients' incision pains. But, it has the advantages of sufficient number of lymph nodes eliminated and easy operation ${ }^{[8]}$.

Liu Baolin ${ }^{[9]}$ applied pulmonary segmentectomy under total thoracoscope in clinical treatment of peripheral early lung cancer and pulmonary metastasis tumor and drew the conclusion that pulmonary segmentectomy under total thoracoscope has high safety and feasibility in treatment of peripheral early lung cancer and pulmonary metastasis tumor. Sun Guogen ${ }^{[10]}$ considered that, thoracoscopic surgery has better curative effect than minimally invasive thoracotomy. The retrospective analysis shows that, the curative effect of pulmonary segmentectomy under total thoracoscope is ideal, and it is highly safe. Besides, it brings fewer complications, and contributes to improving patients' prognosis. There are basically consistent with the research results of the above scholars.

In conclusion, pulmonary segmentectomy under total thoracoscope in clinical treatment of 
peripheral early lung cancer and pulmonary metastasis tumor has high safety and good feasibility, so it deserves to be promoted.

\section{References}

[1] Huang L, Zheng B, Chen C, Zheng W, Zhu Y, Guo C.To Explore Clinical Value of Single-port Video-assisted Thoracoscopic Surgery in Elderly Patients with Non-small Cell Lung Cancer: Lobectomy, Segmentectomy and Lobectomy vs Segmentectomy].Zhongguo Fei Ai Za Zhi. 2018 Apr 20;21(4):287-295.

[2] Pawlak K, Gabryel P, Kujawska A, Kasprzyk M, Piwkowski C, Kuffel B, Dyszkiewicz W.Long-term results of surgical treatment of non-small cell lung cancer in patients over 75 years of age.Kardiochir Torakochirurgia Pol. 2018 Jun;15(2):65-71.

[3] Bamji-Stocke S, van Berkel V, Miller DM, Frieboes HB.A review of metabolism-associated biomarkers in lung cancer diagnosis and treatment.Metabolomics. 2018 Jun;14(6). pii: 81.

[4] Hamaji M, Lee HS, Kawaguchi A, Burt BM.Overall Survival Following Thoracoscopic vs Open Lobectomy for Early-stage Non-small Cell Lung Cancer: A Meta-analysis.Semin Thorac Cardiovasc Surg. 2017 Spring;29(1):104-112. [5] Wang B, Lv F, Zhao L, Du M, Gao S.Video-assisted Thoracoscope versus Video-assisted Mini-thoracotomy for Non-small Cell Lung Cancer: A Meta-analysis].Zhongguo Fei Ai Za Zhi. 2017 May 20;20(5):303-311.

[6] Seguin-Givelet A, Lutz J, Brian E, Grigoroiu M, Gossot D.[Surgical treatment of early stage non-small cell lung cancer by thoracoscopic segmental resection].Rev Mal Respir. 2018 May;35(5):521-530.

[7] $\mathrm{Bu} X \mathrm{X}, \mathrm{Xu} \mathrm{FF,} \mathrm{Ma} \mathrm{J,} \mathrm{Jiang} \mathrm{B.The} \mathrm{expression} \mathrm{of} \mathrm{circulating} \mathrm{tumor} \mathrm{cells} \mathrm{inperipheral} \mathrm{blood} \mathrm{of} \mathrm{patients} \mathrm{with}$ non-small cell lung cancer and its detection.J Biol Regul Homeost Agents. 2018 Jul-Aug;32(4):843-849.

[8] Al-Ameri M, Bergman P, Franco-Cereceda A, Sartipy U.Video-assisted thoracoscopic versus open thoracotomy lobectomy: a Swedish nationwide cohort study.J Thorac Dis. 2018 Jun;10(6):3499-3506.

[9] Dziedzic R, Marjanski T, Binczyk F, Polanska J, Sawicka W, Rzyman W.Favourable outcomes in patients with early-stage non-small-cell lung cancer operated on by video-assisted thoracoscopic surgery: a propensity score-matched analysis.Eur J Cardiothorac Surg. 2018 Mar 14.

[10] Paul S et al. Outcomes after lobectomy using thoracoscopy vs thoracotomy: a comparative effectiveness analysis utilizing the Nationwide Inpatient Sample database.Eur J Cardiothorac Surg. (2013) 